\title{
Original article (short paper) \\ Heart rate as an indicator for exercise prescription for normal, overweight, and obese adolescents
}

\author{
Kamilla Bolonha Gomes \\ Anselmo José Perez \\ Luciana Carletti \\ Universidade Federal do Espírito Santo, Vitória, ES, Brasil \\ Adilson Marques \\ Universidade de Lisboa, Portugal
}

\begin{abstract}
This study aimed to investigate the relationship between heart rate (HR) predicted and $\mathrm{VO}_{2}$ max using a CPET (cardiopulmonary exercise testing) among normal weight, overweight, and obese adolescents. The sample comprised

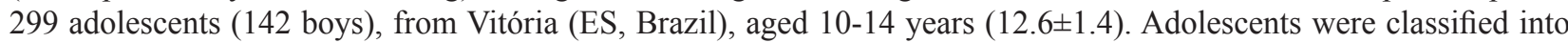
normal weight $(n=236)$, overweight $(n=34)$, and obese $(n=29)$. Adolescents were subjected to a CPET ramp protocol on a treadmill. It was observed that the reserve heart rate (HRres) was similar to the maximum recommended oxygen uptake $\left(\mathrm{VO}_{2}\right)$ for the maintenance and improvement of cardiorespiratory fitness. It was also observed that HRres can be used to control the intensity of exercise sessions in physical education classes independent of weight status. In conclusion, the relationship between the $\mathrm{HR}$ and $\mathrm{VO}_{2}$ for adolescents is linear, allowing the level of intensity to be determined by the HRres proportion related with the correspondent $\% \mathrm{VO}_{2}$ max levels of intensity, according to BMIfor-age categories.
\end{abstract}

Keywords: cardiorespiratory fitness, adolescent, exercise

\section{Introduction}

Youth physical activity participation and physical fitness are associated with a number of health benefits: improvement in bone mineral density, aerobic fitness, muscular strength, endurance, cardiovascular risk factors, and mental health (Janssen \& Leblanc, 2010). Moreover, physical fitness is one of the most important health status markers that predicts cardiovascular disease and mortality (Ortega, Ruiz, Castillo, \& Sjostrom, 2008), mainly among overweight and obese individuals (Cobayashi, Oliveira., Escrivão, Silveira, \& Taddei, 2010; Rodrigues, Perez, Carletti, Bissolli, \& Abreu, 2007). In children and adolescents, physical fitness is associated with improved health-related biomarkers that may further influence young adulthood health (Foley, Quinn, Dwyer, Venn, \& Jones, 2008; Simmons, Griffin, Steele, Wareham, \& Ekelund, 2008).

As the health of young people is gaining attention, due to the global epidemic of obesity during childhood (Onis, Blossner, \& Borghi, 2010), physical activity recommendations have been highlighted (World Health Organization, 2010) to prevent health problems (Garcia-Hermoso, Saavedra, Escalante, SanchezLopez, \& Martinez-Vizcaino, 2014; Prado et al., 2014), improve health (Ortega et al., 2008), and increase physical fitness levels (Strong et al., 2005). Thus, safe and effective exercise prescription for young people is a growing concern because it is important that the exercise be at the appropriate frequency, duration and intensity.
Regardless of age, the intensity of physical activity is usually defined in terms of percentages of maximal oxygen uptake $\left(\% \mathrm{VO}_{2} \max \right)$, maximal heart rate $(\% \mathrm{HRmax})$ and reserve heart rate (\%HRres), or as a multiple of resting metabolic rate (1 MET) (Garber et al., 2011). Although the $\% \mathrm{VO}_{2} \max$ is the gold standard to determine exercise intensity, its measurement is still costly and and not easily available since a cardiopulmonary exercise testing (CPET) is required. These difficulties make the HR a an alternative option to measure exercise intensity. There is a linear relationship between $\mathrm{VO}_{2}$ max and HRmax at submaximal exercise intensities (Garber et al., 2011).

The American College of Sports Medicine has recommended that the appropriate exercise intensity for improving cardiorespiratory fitness for adults is $50-85 \%$ of $\mathrm{VO}_{2} \max , 60-90 \%$ of age-predicted HRmax (American College of Sports Medicine, 2013). The equivalency between $\mathrm{HR}$ and $\mathrm{VO}_{2}$ is based on the fact that $\% \mathrm{VO}_{2}$ max or $\% \mathrm{VO}_{2}$ res equals the same $\% \mathrm{HRmax}$ or \%HRres. This was demonstrated by Swain and Leutholtz (1997), who observed that HRres was equivalent to $\mathrm{VO}_{2}$ res. In fact, the relationship between $\mathrm{VO}_{2}$ and $\mathrm{HR}$ during rest and exercise is well documented for adults (Cunha, Midgley, Monteiro, \& Farinatti, 2010; Swain \& Franklin, 2002; Swain \& Leutholtz, 1997; Swain, Leutholtz, King, Haas, \& Branch, 1998), and extensively used for exercise prescription (ACSM, 2013). Nonetheless, there is limited research and evidence regarding the relationship between $\mathrm{VO}_{2}$ and $\mathrm{HR}$ in children and adolescents (Ekelund et al., 2001; Hui \& Chan, 2006). Children and 
adolescents are physiologically different from adults and the association between $\mathrm{VO}_{2}$ and $\mathrm{HR}$ might not be similar to the association known in adults, as observed by Hui and Chan (2006). Furthermore, there is no evidence of the influence of BMI categories on children and adolescents physical performance.

Establishing an objective criterion to prescribe children and adolescents exercise has practical implications for their health promotion. Exercise intensity recommended for children and adolescents needs further evaluation. Therefore, this study aimed to investigate and determine the relationship between HR predicted and $\mathrm{VO}_{2}$ max using a CPET among normal weight, overweight, and obese adolescents.

\section{Methods}

\section{Participants}

The present study used data of students from public and private schools in the 7 regions of Vitória municipality (ES, Brazil), aged 10-14 years (12.6 \pm 1.4$)$. Data from a proportionate stratified random sample of students were collected as previously reported (Rodrigues, Perez, Carletti, Bissoli \& Abreu, 2006), taking into account the location and number of students, by gender, in each school. All students from 10 to 14 years of age with a health status that allowed participation in physical education classes were eligible. Data from 380 (177 boys, 203 girls) out of 27491 students were collected. Of these 380 participants, 299 (142 boys, 157 girls) attended all inclusion criteria of CPET protocol. During physical education classes students were taken to the laboratory by social worker, or were accompanied by their parents. In the laboratory they were received by the research team conducting the CPET. Prior to testing, the participants and their guardians were informed about the objectives of the study, procedures, benefits, and probable risks of the investigation. Written informed consent was then obtained from the guardians. The Faculdade Salesiana de Vitória (ES, Brazil) Ethics Committee approved the study protocol, and the study was conducted according to ethical standards in sport and exercise science research (Harriss \& Atkinson, 2013).

Experimental design:

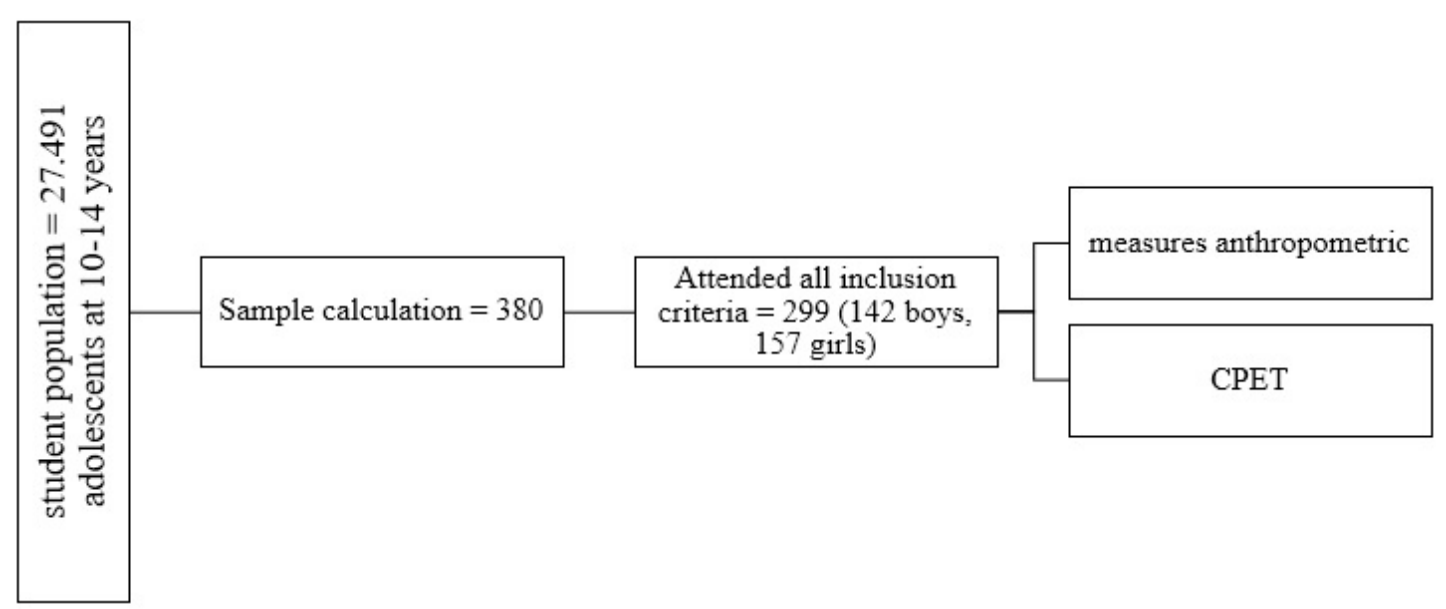

\section{Measures Anthropometric}

To assess weight status, participants were weighed to the nearest $0.1 \mathrm{~kg}$ wearing minimal clothes and without shoes, using an electronic scale (Welmy, model RW200; São Paulo, BR). Height was measured with a stadiometer to the nearest $0.1 \mathrm{~cm}$. Body mass index was then calculated based on mass (kilograms) divided by height (square meters). Participants were classified into normal weight (NW, percentile $<85, \mathrm{n}=236$ ), overweight ( $\mathrm{OW}$, percentile $>$ $85 \leq 97, n=34)$, and obese (OB, percentile $>97, n=29)$ categories according to age- and gender-specific cut-off points proposed by World Health Organization (Onis et $a l ., 2010)$.

\section{Cardiopulmonary exercise testing protocol}

Prior to CPET, participants were instructed on exercise testing procedures: how to walk and run on a treadmill, and then engaged in a standardized 2 minutes warm-up period. Participants walked and ran on a calibrated motor-driven treadmill (Inbrasport Super ATL, RGS, Brazil), according to an incremental exercise protocol (Rodrigues, Perez, Carletti, Bissoli, \& Abreu, 2006). The test protocol started at $5 \mathrm{~km} / \mathrm{h}$ with an inclination of $0.0 \%$. The treadmill was then increased to the participants' comfortable running speed, to a maximum of $12 \mathrm{~km} / \mathrm{h}$; the inclination was raised until $12 \%$ was reached as described previously (Rodrigues, Bissoli, Pires, \& Abreu, 2004) using an individualized protocol (Karila, de Blic, Waernessyckle, 
Benoist, \& Scheinmann, 2001). $\mathrm{VO}_{2}$ and carbon dioxide $\left(\mathrm{V}_{\mathrm{CO} 2}\right)$ output were measured using a breath-by-breath analyser (CPX/D system; Medical Graphics Corporation, St Paul, MN, USA). The system also provided information about pulmonary ventilation, ventilator equivalent of oxygen, and respiratory exchange ratio. The equipment was calibrated before each participant's test, and parts of bare skin in contact with the equipment were sanitised before use. To consider whether the $\mathrm{VO}_{2} \max$ was reached, at least three of the following criteria were observed (Karila et al., 2001): 1) failure to maintain the required speed; 2) respiratory exchange ratio $>1.0 ; 3$ ) HR within 5 beats/min of the age-predicted HRmax; 4) $\mathrm{VO}_{2} \max >85 \%$ of predicted. Resting HR (HRresting) determination was obtained from a resting 12-lead electrocardiogram (ECG) (CardioPerfect RMD) connected to the equipment (CPX/D system; Medical Graphics Corporation, St Paul, MN, USA). Participants remained in the supine position for approximately five minutes, and the minimum HR was registered (HRresting). The test was performed at least 2 hours after the last meal. The lab temperature was maintained between 20 and 23 degrees Celsius, and there was equipment and pharmaceuticals for unforeseen cases with the participants.

\section{Data analysis}

HRres was obtained from the subtraction of HRmax measured in the CPET by HRresting (HRmax - HRresting). The HRres was then multiplied by the percentages 50, 60, 70, 80, and 90. From the values of HRmax, the predicted HR was calculated by the simple method (\%HRmax): HRmax measure multiplied by the same percentage of HRres. These predicted values were then compared with the experimental results from CPET in the percentages of 50, 60, 70, 80 and 90 of $\mathrm{VO}_{2} \max$. Descriptive statistics were calculated (means, standard deviation) for all variables. Variables were examined for normality and homoscedasticity with Kolmogorov-Smirnov and Levene's tests. One-way ANOVA followed by Tukey's post hoc test, and student's t-test, were used to assess the differences among BMI categories and between sexes. The Pearson correlation was used to verify the relationship between absolute HR (measured and predicted) values in percentages of $\mathrm{VO}_{2}$ max in the three groups of adolescents. All statistical analyses were performed using SigmaStat 3.5. A value of $p<0.05$ was considered significant.

\section{Results}

Characteristics of the participants according BMI categories are presented in Table 1.

The measured and predicted HR (beat. $\mathrm{min}^{-1}$ ) results in different percentages of $\mathrm{VO}_{2} \max$ intensity are presented in Table 2. For the three BMI categories, in both sexes, HR assessed by the simple method (\% HRmax) was significantly lower than the HRres and HR measured during the CPET in all percentages of $\mathrm{VO}_{2}$ analysed. The average HRresting used to compute HRres was $76 \pm 9$ beats/min and $82 \pm 10$ beats $/ \mathrm{min}$ $(p<0.05)$ for NW, $78 \pm 9$ beats/min and $83 \pm 11$ beats/min for OW, $86 \pm 14$ beats/min and $90 \pm 11$ beats/min for OB participants. Significant differences between OB participants and the other BMI categories (NW and OW) were observed. For HRmax in the CPET there were no significant differences among BMI categories $(\mathrm{NW}=199 \pm 8$ beats $/ \mathrm{min}, \mathrm{OW}=200 \pm 8$ beats $/ \mathrm{min}$, $\mathrm{OB}=199 \pm 9$ beats/min).

The correlation between measured HR vs. predicted method HRres, and HR vs. predicted simple method (\%HRmax), in normal weight, overweight, and obese adolescents, are shown in Figures 1, 2 and 3 . There was a high correlation among measured $\mathrm{HR}$ in all percentiles of $\mathrm{VO}_{2} \max (50,60,70,80$ and $90 \%)$ with method HRres and \%HRmax. This occurred for the three groups in both sexes. The medium and strong correlation values range from 0.50 to 0.80 of the three groups: normal weight, overweight and obesity, involving all percentages studied.

For the $\mathrm{VO}_{2}$ max directly measured in the CPET among NW participants, boys had significantly higher values than girls (48.2 \pm 7.2 vs $39.1 \pm 6.0 \mathrm{ml} . \mathrm{kg} / \mathrm{min} ; p=0.001)$. For OW, boys also had a significantly higher value for $\mathrm{VO}_{2}$ max than girls $(41.1 \pm 6.5$ vs $35.3 \pm 5.1 \mathrm{ml} . \mathrm{kg} / \mathrm{min} ; p=0.006)$. Nonetheless, among OB participants there was no significant difference between boys and girls (33.4 \pm 5.6 vs $32.1 \pm 4.4 \mathrm{ml} . \mathrm{kg} / \mathrm{min} ; \mathrm{p}=0.539)$.

Results of the $\% \mathrm{HR}$ for percentages of $\mathrm{VO}_{2}$ max are presented in Table 3. For all levels of $\mathrm{VO}_{2}$ max intensity, gender, and BMI categories, no significant differences were observed.

For HRres, results are shown in Table 4. It is possible to observe that $\mathrm{OB}$ boys and girls had significantly higher percentages of HRres than NW boys and girls for all $\% \mathrm{VO}_{2}$ max levels of intensity. Comparing boys and girls, differences between sexes were only observed for the NW, girls had lowers HRres for all $\% \mathrm{VO}_{2}$ max levels of intensity.

Table 1. Anthropometric characteristics of participants aged 10-14 years.

\begin{tabular}{|c|c|c|c|}
\hline & $\begin{array}{c}\text { NW } \\
(\mathbf{n}=\mathbf{2 3 6})\end{array}$ & $\begin{array}{c}\text { OW } \\
(n=34)\end{array}$ & $\begin{array}{c}\text { OB } \\
(n=29) \\
\end{array}$ \\
\hline Age & $12.7 \pm 1.4$ & $12.5 \pm 1.5$ & $12.4 \pm 1.2$ \\
\hline Weight (kg) & $40.6 \pm 9$ & $54.2 \pm 10^{*}$ & $65.8 \pm 12.5^{\text {t\# }}$ \\
\hline Height (m) & $1.52 \pm 0.11$ & $1.55 \pm 0.1$ & $1.57 \pm 0.09^{\dagger}$ \\
\hline BMI $\left(\mathrm{kg} / \mathrm{m}^{2}\right)$ & $17.5 \pm 2.0$ & $22.5 \pm 1.7 *$ & $26.5 \pm 3.4^{\dagger \#}$ \\
\hline
\end{tabular}

$p<0.05:{ }^{*} \mathrm{NW}$ vs. OW; ${ }^{\dagger} \mathrm{NW}$ vs. OB; ${ }^{\sharp} \mathrm{OW}$ vs. OB.

$\mathrm{NW}$, normal weight; OW, overweight; OB, obese; BMI, body mass index

Tests by ANOVA followed by Tukey's post hoc test. 
Tables 2. HR (beats/min) values according percentages of $\mathrm{VO}_{2} \mathrm{max}$ in normal weight, overweight, and obese adolescents

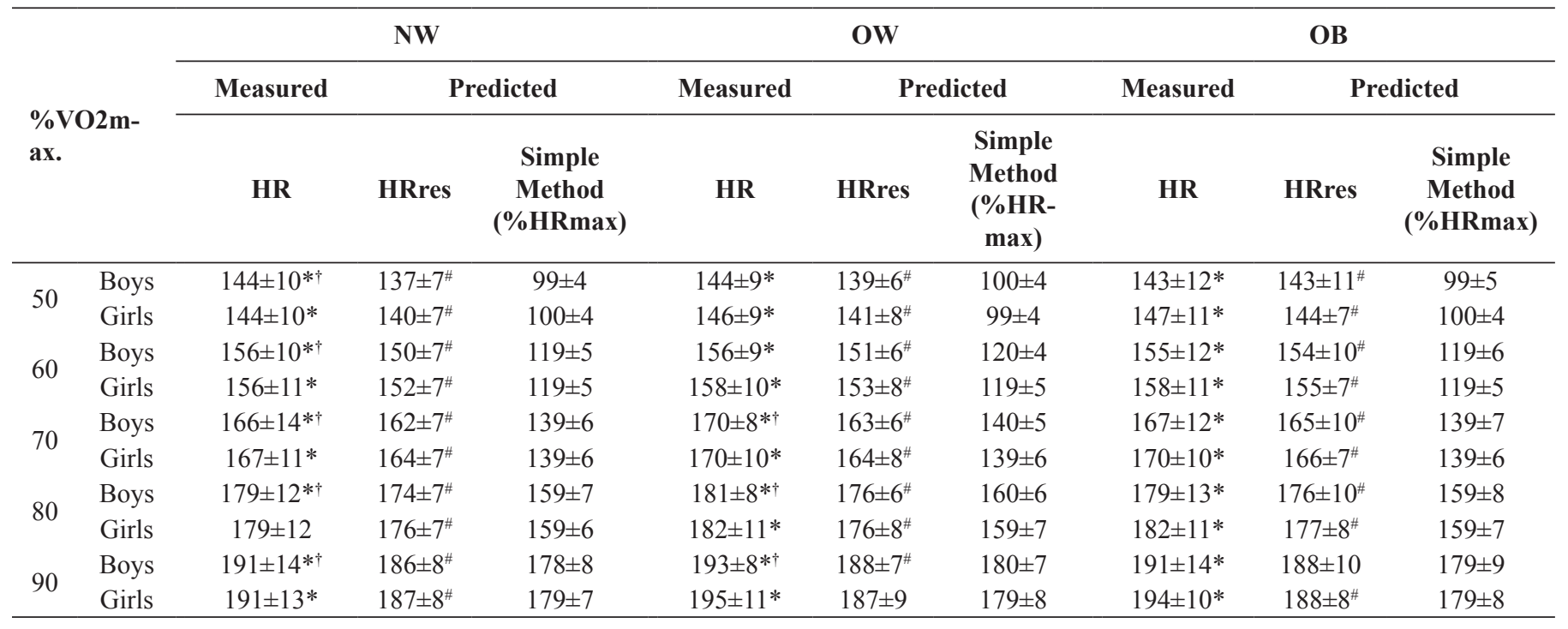

p $<0.05: *$ HR vs. Simple method HRmax; † HR vs. Simple method HRres; \# HRres vs. Simple method HRmax

NW, normal weight; OW, overweight; OB, obese; BMI, body mass; HR, heart rate; HRres, reserve heart rate; HRmax, maximum heart rate.

Tested by ANOVA followed by Tukey's post hoc test

Tables 3. Percentages of measured HR according to percentages of $\mathrm{VO}_{2}$ max in normal weight, overweight, and obese adolescents.

\begin{tabular}{lcccc}
\hline \multicolumn{2}{c}{ \%VO $\mathbf{m a x}$} & NW (\%) & OW (\%) & OB (\%) \\
\hline $50 \%$ & Boys & $72.6 \pm 4.6$ & $72.0 \pm 3.0$ & $71.7 \pm 4.0$ \\
& Girls & $72.5 \pm 4.7$ & $73.4 \pm 2.6$ & $73.7 \pm 4.2$ \\
$60 \%$ & Boys & $78.5 \pm 4.3$ & $78.2 \pm 2.7$ & $77.8 \pm 3.6$ \\
& Girls & $78.3 \pm 4.4$ & $79.5 \pm 2.8$ & $79.4 \pm 3.5$ \\
$70 \%$ & Boys & $84.4 \pm 4.4$ & $84.8 \pm 2.2$ & $83.8 \pm 3.7$ \\
& Girls & $84.1 \pm 4.4$ & $85.5 \pm 2.4$ & $85.6 \pm 3.0$ \\
$80 \%$ & Boys & $90.3 \pm 4.8$ & $90.7 \pm 2.2$ & $90.1 \pm 4.0$ \\
& Girls & $89.9 \pm 4.8$ & $91.4 \pm 2.6$ & $91.4 \pm 2.8$ \\
$90 \%$ & Boys & $96.3 \pm 5.5$ & $96.7 \pm 2.5$ & $95.8 \pm 4.2$ \\
& Girls & $95.7 \pm 5.2$ & $98.0 \pm 2.9$ & $97.3 \pm 2.9$ \\
\hline
\end{tabular}

NW, normal weight; OW, overweight; OB, obese

Tested by ANOVA.

Table 4. HRres according to percentages of $\mathrm{VO}_{2} \max$ in normal weight, overweight, and obese adolescents.

\begin{tabular}{ccccc}
\hline \multicolumn{2}{c}{$\mathbf{\% V O}_{\mathbf{2}} \mathbf{m a x}$} & $\mathbf{N W}(\mathbf{\%})$ & OW (\%) & OB (\%) \\
\hline $50 \%$ & Boys & $69.3 \pm 2.4$ & $69.5 \pm 2.5$ & $71.7 \pm 3.1^{* \#}$ \\
& Girls & $70.5 \pm 2.4^{* *}$ & $70.8 \pm 2.5$ & $72.5 \pm 2.9^{*}$ \\
$60 \%$ & Boys & $75.4 \pm 1.9$ & $75.6 \pm 2.0$ & $77.4 \pm 2.5^{* \#}$ \\
& Girls & $76.4 \pm 1.9^{* *}$ & $76.7 \pm 2.0$ & $78.0 \pm 2.3^{*}$ \\
$70 \%$ & Boys & $81.6 \pm 1.5$ & $81.7 \pm 1.5$ & $83.0 \pm 1.9^{* \#}$ \\
& Girls & $82.3 \pm 1.4^{* *}$ & $82.5 \pm 1.5$ & $83.5 \pm 1.7^{*}$ \\
$80 \%$ & Boys & $87.7 \pm 1.0$ & $87.8 \pm 1.0$ & $88.7 \pm 1.3^{* \#}$ \\
& Girls & $88.2 \pm 1.0^{* *}$ & $88.3 \pm 1.0$ & $89.0 \pm 1.2^{*}$ \\
$90 \%$ & Boys & $93.9 \pm 0.5$ & $93.9 \pm 0.5$ & $94.3 \pm 0.6^{* \#}$ \\
& Girls & $94.1 \pm 0.5^{* *}$ & $94.2 \pm 0.5$ & $94.5 \pm 0.6^{*}$ \\
\hline
\end{tabular}

$p<0.05: * \mathrm{NW}$ vs. $\mathrm{OB} ;{ }^{\dagger} \mathrm{NW}$ vs. OW; ${ }^{*} \mathrm{OW}$ vs. OB; **boys vs. girls.

$\mathrm{NW}$, normal weight; OW, overweight; OB, obese

ANOVA followed by Tukey's post hoc test was used to test differences among BMI categories.

Student t-test was used to test differences between sexes. 


\section{Normal Weight}

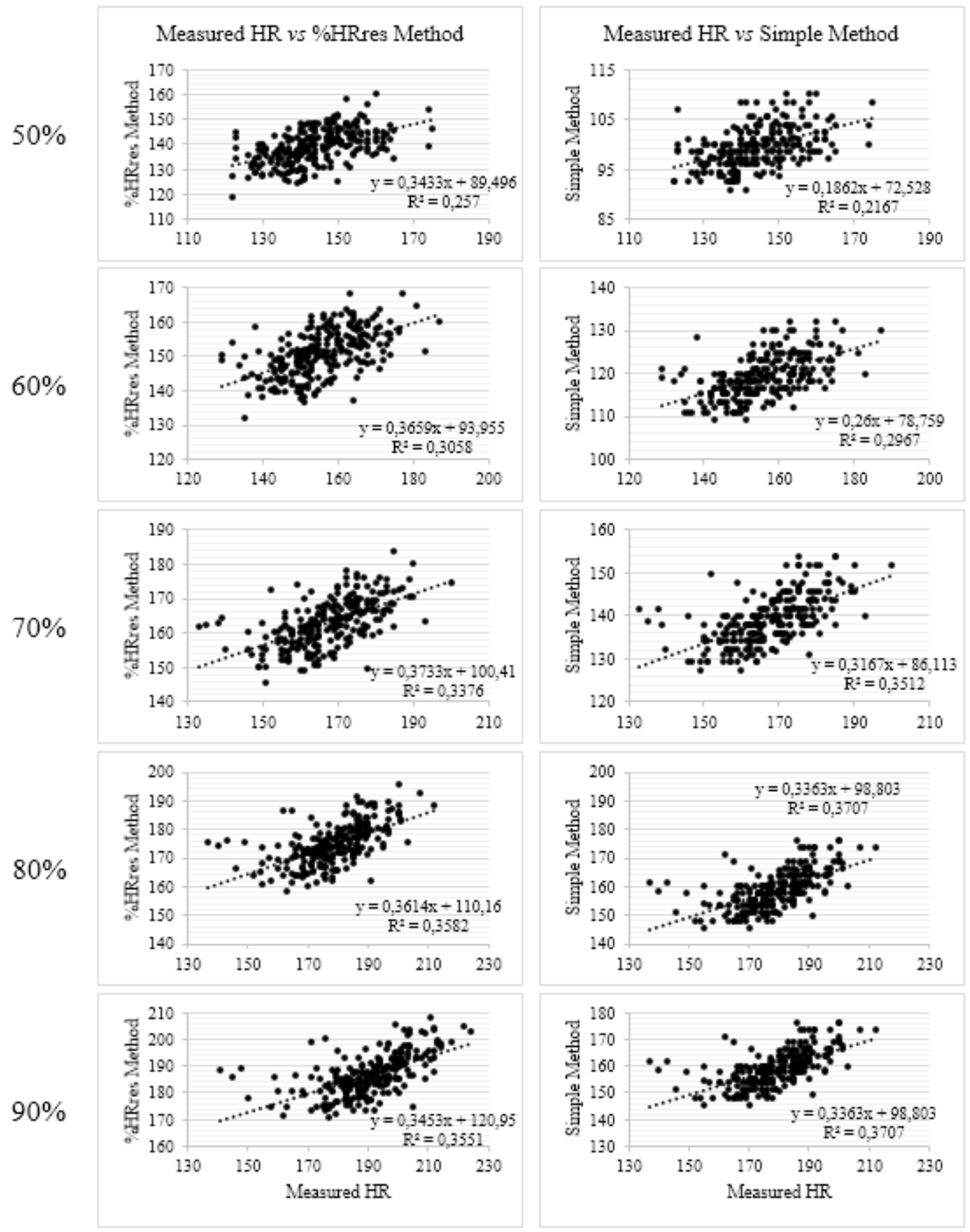

Figure 1. Correlation between measured HR vs. predicted \%HRres method and measured HR vs predicted simple method (HRmax) in normal weight for both sexes together. 


\section{Overweight}

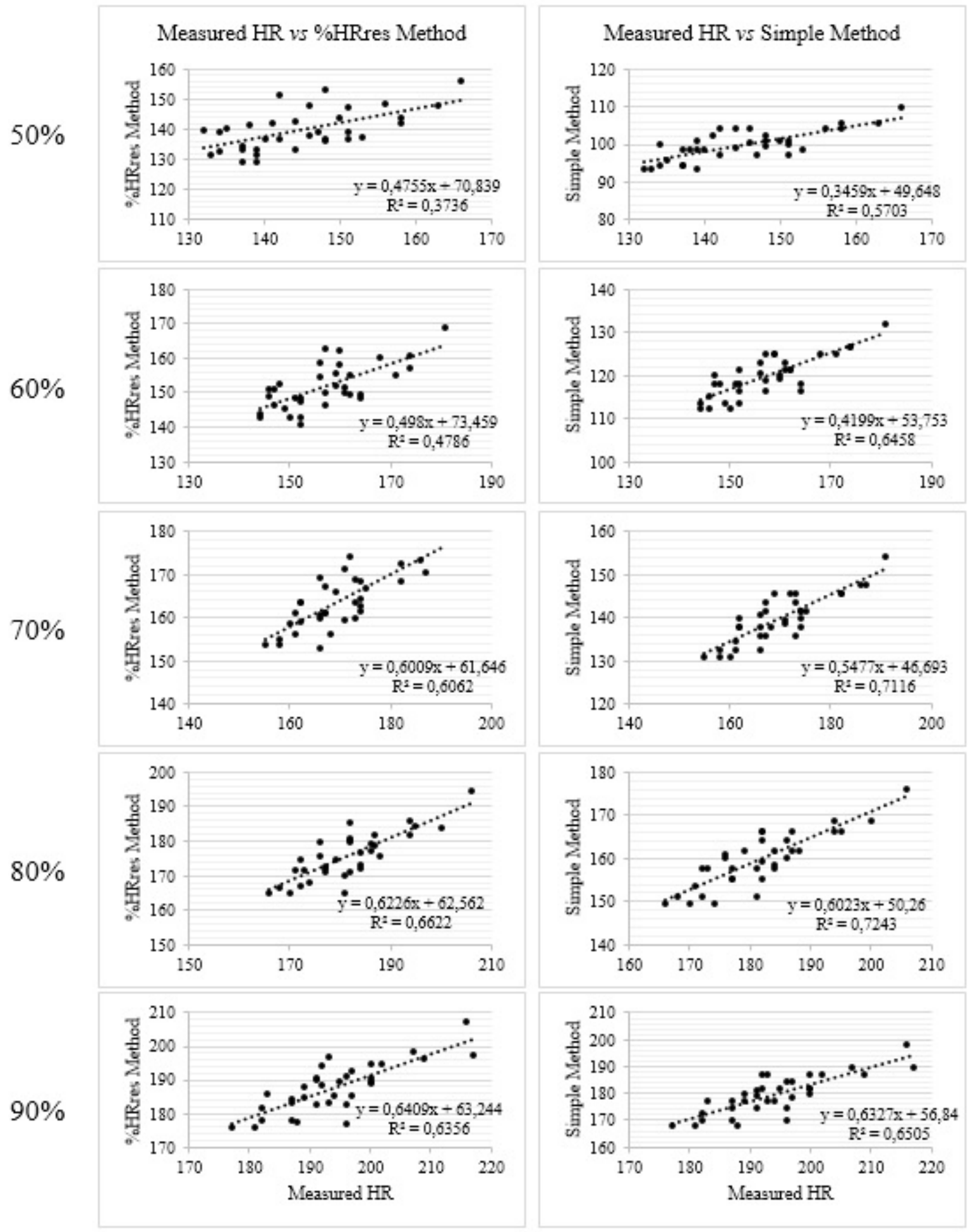

Figure 2. Correlation between measured HR vs. predicted \%HRres method and measured HR vs predicted simple method (HRmax) in overweight in both sexes together. 


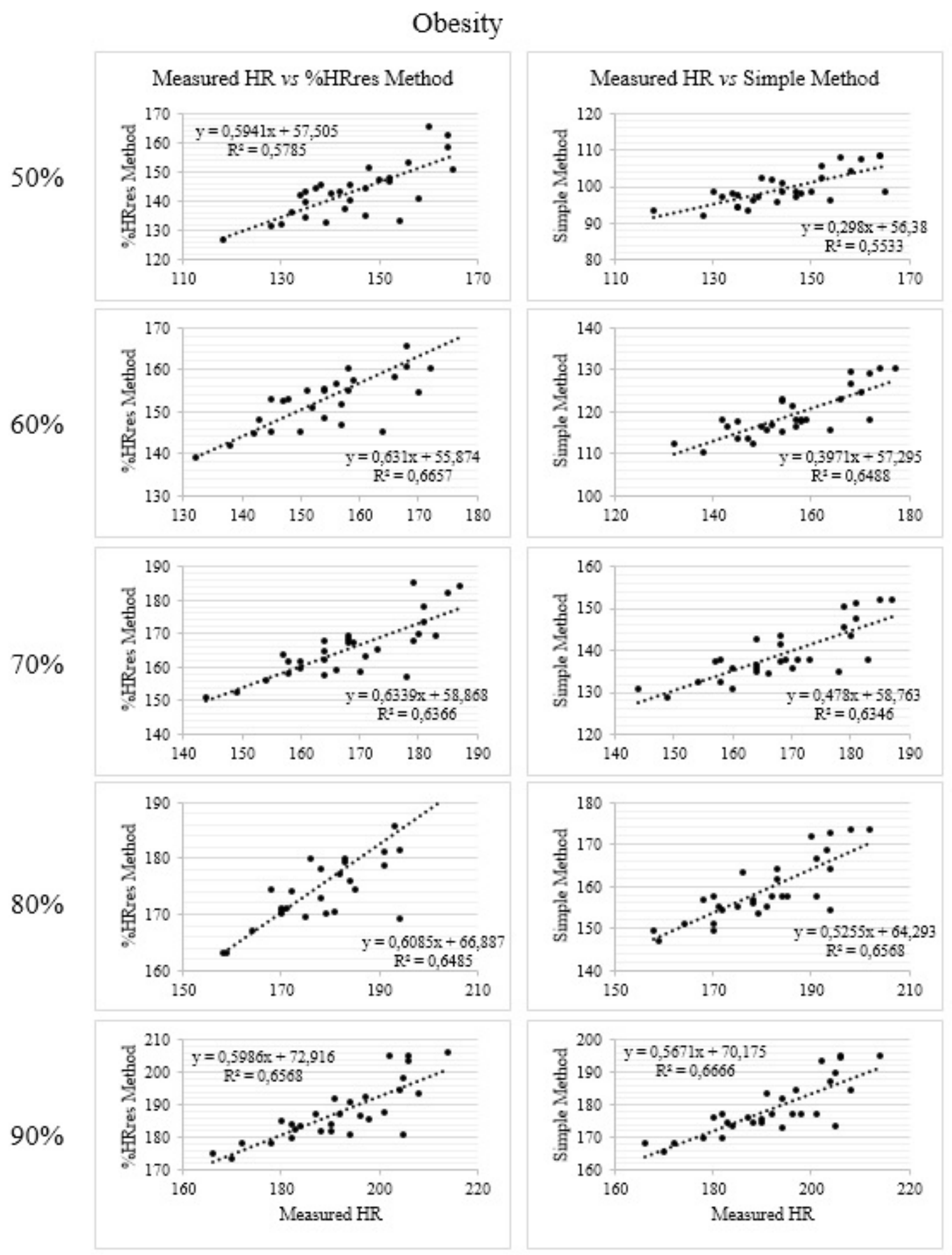

Figure 3. Correlation between measured HR vs. predicted \%HRres method and measured HR vs predicted simple method (HRmax) in obesity in both genders together. 


\section{Discussion}

It was observed that HRres were similar to the maximum recommended $\mathrm{VO}_{2}$ for the maintenance and improvement of cardiorespiratory fitness for children and adolescents aged 1014 years old. It was also observed that HRres can be used to control the intensity of exercise sessions in physical education classes for normal weight, overweight, and obese adolescents. This is considered a relatively inexpensive and easy way to allow teachers to know the higher \%HRres necessary for obese adolescents.

For adults, the cardiorespiratory exercise recommendation is already defined (Cunha et al., 2010; Garber et al., 2011). To our knowledge there is no data for adolescents. Even though the values presented in this study are not in accordance with previous studies (Ekelund et al., 2001; Hui \& Chan, 2006), a similar linear relationship between the $\mathrm{HR}$ and $\mathrm{VO}_{2}$ during CPET was observed, approving the use of HR as a valid parameter to estimate intensity in adolescents' physical activity. The differences between the studies can be explained by the characteristics of the sample (e.g. age average, weight, height) and the values of HRresting. For example, for the present study the HR used for the HRres calculation was the lowest registered by the ECG, after a 5-minute of resting, and not a baseline heart rate (registered when waking up).

According to Epstein et al. (2001), HRresting changes with age and FCmax is close to 200 beats/min during adolescence, showing consistency with the results found for HRmax in the three BMI categories. Despite not being an objective of this study (comparing these variables between ages of 10 to 14 years), it is worth emphasizing that HRres takes into consideration changes in HRresting and HRmax. This reinforces the importance of performing the CPET to identify the maximum measured HR in effort. Epstein et al. (2001) stated that the ACSM recommendations for adolescents may differ from the adult population with respect to intensity, duration/amount. This supports the need to understand the most suitable level of exercise intensity for adolescents. It is important to mention that the studies previously referred to are based on normal weight adolescents, since studies with overweight adolescents are scarce (Ekelund et al., 2001; Hui \& Chan, 2006).

In obese adults, the study performed by Pinet, Prud'homme, Gallant and Boulay (2008) suggests the use of HRres when it is not possible to assess oxygen consumption. This information is important because CPET method is not easily accessible to all populations, and has high costs of implementation, leaving HR as a valid and relatively simple variable which can be easily measured at a low cost. Thus, our findings suggest applying different recommendations of exercise for adolescents with distinct BMI categories, using the HRmax scale in CPET.

Even though we have identified correlation of medium intensity and good coefficient of correlation between HR predictive methods (HRres and HRmax), the values found in the $\%$ HRmax underestimate the amounts measured. Consequently, whether these results are to be used for exercise prescription, we would be underestimating the intensity of the effort, which is not in accordance with the ACSM recommendations (ACSM,
2013). However, these results differ when equations are used to predict HRmax (Cunha et al., 2010) as previously demonstrated by Caputo, Cozzensa da Silva and Rombaldi (2012), in which the authors recommend avoiding the equations because they restrict the HRmax. So far, there is no appropriate formula for this estimation. Therefore, by choosing to use equations to calculate HRmax, and \%HRmax, there is a risk of erroneous conclusions regarding the level of intensity to apply when prescribing exercise.

Using the data collected in this study, it is possible to identify more precisely, and at a low cost, benefits for adolescents, considering the relationship between the HRres and the length of the aerobic activity ( $\geq 30$ minutes per session, above $50 \%$ HRres) suggested in exercise programs (Epstein et al., 2001), and the importance of using percentages (Ekelund et al., 2001). In addition, the sample size and the techniques used in this study ensure the importance of the measure of the HRres.

In summary, it was observed that the relationship between the $\mathrm{HR}$ and $\mathrm{VO}_{2}$ in CPET for adolescents aged 10-14 years is linear, for both sexes, allowing the level of intensity to be determined by the HRres proportion related with the correspondent $\% \mathrm{VO}_{2}$ max levels of intensity according to BMI-for-age categories. HRres might be the most suitable parameter to prescribe exercises for adolescents, particularly overweight ones, because it is closer to the $\mathrm{VO}_{2}$ max intensity analysed, determining the appropriate aerobic stimuli to allow better performance of the cardiovascular system within this specific population.

Some limitations warrant mention when interpreting the findings from this study. Biological maturation was not assessed; the process of growth and development might influence the findings. The combination of sample size in general and the overweight and obese adolescents in particular may limit generalization of the results. Keeping all adolescents engaged and motivated during the tests, despite the instructors' efforts, was also one of the constraints identified during this study.

\section{References}

ACSM. (2013). ACSM's guidelines for exercise testing and prescription. Baltimore: Lippincott Williams \& Wilkins.

Caputo, E., Cozzensa da Silva, M., \& Rombaldi, A. (2012). Comparação da frequência cardíaca máxima obtida por diferentes métodos. Revista de Educação Física/UEM, 23(2), 277-284.

Cobayashi, F., Oliveira., F., Escrivão, M., Silveira, D., \& Taddei, J. (2010). Obesidade e fatores de risco cardiovascular em adolescentes de escolas públicas. Arquivos Brasileiros de Cardiologia, 95(2), 200-206.

Cunha, F.A., Midgley, A.W., Monteiro, W.D., \& Farinatti, P.T. (2010). Influence of cardiopulmonary exercise testing protocol and resting $\mathrm{VO}(2)$ assessment on \%HR( $\max ), \% \mathrm{HRR}, \% \mathrm{VO}(2 \max )$ and $\% \mathrm{VO}(2) \mathrm{R}$ relationships. International Journal of Sports Medicine, 31(5), 319-326.

Ekelund, U., Poortvliet, E., Yngve, A., Hurtig-Wennlov, A., Nilsson, A., \& Sjostrom, M. (2001). Heart rate as an indicator of the intensity of physical activity in human adolescents. European Journal of Applied Physiology, 85(3-4), 244-249. 
Epstein, L.H., Paluch, R.A., Kalakanis, L.E., Goldfield, G.S., Cerny, F.J., \& Roemmich, J.N. (2001). How much activity do youth get? A quantitative review of heart-rate measured activity. Pediatrics, 108(3).

Foley, S., Quinn, S., Dwyer, T., Venn, A., \& Jones, G. (2008). Measures of childhood fitness and body mass index are associated with bone mass in adulthood: a 20-year prospective study. Journal of Bone and Mineral Research, 23(7), 994-1001.

Garber, C.E., Blissmer, B., Deschenes, M.R., Franklin, B.A., Lamonte, M.J., Lee, I.M., Nieman, D.C., Swain, D.P. (2011). American College of Sports Medicine position stand. Quantity and quality of exercise for developing and maintaining cardiorespiratory, musculoskeletal, and neuromotor fitness in apparently healthy adults: guidance for prescribing exercise. Medicine and Science in Sports and Exercise, 43(7), 1334-1359.

Garcia-Hermoso, A., Saavedra, J.M., Escalante, Y., Sanchez-Lopez, M., \& Martinez-Vizcaino, V. (2014). Endocrinology and Adolescence: aerobic exercise reduces insulin resistance markers in obese youth: a meta-analysis of randomized controlled trials. European Journal of Endocrinology / European Federation of Endocrine Societies, 171(4), R163-171.

Harriss, D.J., \& Atkinson, G. (2013). Ethical standards in sport and exercise science research: 2014 update. International Journal of Sports Medicine, 34(12), 1025-1028.

Hui, S.S.C., \& Chan, J.W.S. (2006). The relationship between heart rate reserve and oxygen uptake reserve in children and adolescents. Research Quarterly for Exercise and Sport, 77(1), 41-49.

Janssen, I., \& Leblanc, A.G. (2010). Systematic review of the health benefits of physical activity and fitness in school-aged children and youth. Int J Behav Nutr Phys Act, 7, 40.

Karila, C., de Blic, J., Waernessyckle, S., Benoist, M.R., \& Scheinmann, P. (2001). Cardiopulmonary exercise testing in children: an individualized protocol for workload increase. Chest, 120(1), 81-87.

Onis, M., Blossner, M., \& Borghi, E. (2010). Global prevalence and trends of overweight and obesity among preschool children. American Journal of Clinical Nutrition, 92(5), 1257-1264.

Ortega, F.B., Ruiz, J.R., Castillo, M.J., \& Sjostrom, M. (2008). Physical fitness in childhood and adolescence: a powerful marker of health. International Journal of Obesity, 32(1), 1-11.

Pinet, B.M., Prud'homme, D., Gallant, C.A., \& Boulay, P. (2008). Exercise intensity prescription in obese individuals. Obesity, 16(9), 2088-2095.

Prado, W.L., Balagopal, P.B., Lofrano-Prado, M.C., Oyama, L.M., Tenorio, T.R., Botero, J.P., Hill, J.O. (2014). Effect of aerobic exercise on hunger feelings and satiety regulating hormones in obese teenage girls. Pediatric Exercise Science, 26(4), 463-469.

Rodrigues, A.N., Bissoli, N.S., Pires, J.G.P., \& Abreu, G.R. (2004). A populational study on cariorespiratory and metabolic parameters in schoolchildren of Vitoria, Brazil: Preliminary results. Journal of Hypertension, 22, S51-S51.

Rodrigues, A.N., Perez, A.J., Carletti, L., Bissoli, N.S., \& Abreu, G.R. (2006). Maximum oxygen uptake in adolescents as measured by cardiopulmonary exercise testing: a classification proposal. Jornal de Pediatria, 82(6), 426-430.

Rodrigues, A.N., Perez, A.J., Carletti, L., Bissolli, N.S., \& Abreu, G.R. (2007). The association between cardiorespiratory fitness and cardiovascular risk in adolescents. Jornal de Pediatria, 83(5), 429-435.

Simmons, R.K., Griffin, S.J., Steele, R., Wareham, N.J., \& Ekelund, U. (2008). Increasing overall physical activity and aerobic fitness is associated with improvements in metabolic risk: cohort analysis of the ProActive trial. Diab tologia, 51(5), 787-794.

Strong, W.B., Malina, R.M., Blimkie, C.J., Daniels, S.R., Dishman, R.K., Gutin, B. Hergenroeder, A.C., Must, A., Nixon, P.A., Pivarnik, J.M., Rowland, T., Trost, S., Trudeau, F. (2005). Evidence based physical activity for school-age youth. Journal of Pediatrics, 146(6), 732-737.

Swain, D.P., \& Franklin, B.A. (2002). VO(2) reserve and the minimal intensity for improving cardiorespiratory fitness. Medicine and Science in Sports and Exercise, 34(1), 152-157.

Swain, D.P., \& Leutholtz, B.C. (1997). Heart rate reserve is equivalent to $\% \mathrm{VO} 2$ reserve, not to $\% \mathrm{VO} 2 \mathrm{max}$. Medicine and Science in Sports and Exercise, 29(3), 410-414.

Swain, D.P., Leutholtz, B.C., King, M.E., Haas, L.A., \& Branch, J.D. (1998). Relationship between \% heart rate reserve and \% VO2 reserve in treadmill exercise. Medicine and Science in Sports and Exercise, 30(2), 318-321.

World Health Organization, WHO. (2010). Global recommendations on physical activity for health. WHO Press: Geneva, Switzerland.

\section{Autor's note}

Kamilla Bolonha Gomes, Anselmo José Perez, Luciana Carletti are affiliated with the Federal University of Espírito Santo, Exercise Physiology Laboratory.

Adilson Marques is affiliated with the University of Lisboa, Interdisciplinary Centre for Human Performance Study.

\section{Corresponding author}

Kamilla Bolonha Gomes

Rua Vicente de Oliveira, 25, Vitória, ES, Brazil.

Email: kamilla.bolonha@outlook.com

Manuscript received on June 09, 2015

Manuscript accepted on January 20, 2016

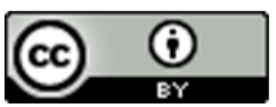

Motriz. The Journal of Physical Education. UNESP. Rio Claro, SP, Brazil - eISSN: 1980-6574 - under a license Creative Commons - Version 3.0 